\title{
Nanostructuring as a procedure to control the field dependence of the magnetocaloric effect
}

\author{
D. Doblas ${ }^{\mathrm{a}, 1}$, L.M. Moreno-Ramírez ${ }^{\text {a }}$, V. Franco ${ }^{\mathrm{a}, *}$, A. Conde $^{\mathrm{a}}$, A.V. Svalov ${ }^{\mathrm{b}, \mathrm{c}}$, G.V. Kurlyandskaya ${ }^{\text {c,d }}$ \\ ${ }^{a}$ Dpto. Física de la Materia Condensada, ICMSE-CSIC, Universidad de Sevilla, P.O. Box 1065, 41080 Sevilla, Spain

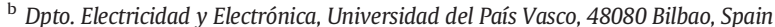 \\ c Ural Federal University, 620083 Ekaterinburg, Russia \\ ¿ BCMaterials, Universidad del País Vasco (UPV/EHU), Leioa, Spain
}

\section{H I G H L I G H T S}

- The MCE of Gd/Ti multilayers and its field dependence has been studied.

- Nanostructuring enhances the field responsiveness of MCE near $\mathrm{T}_{\mathrm{c}}$.

- Curie temperature distributions due to finite size scaling are responsible for this.

- A combination of numerical simulations and experimental results are presented.

- This is a possibility for improving MCE response at the nanoscale.

\section{G R A P H I C A L A B S T R A C T}

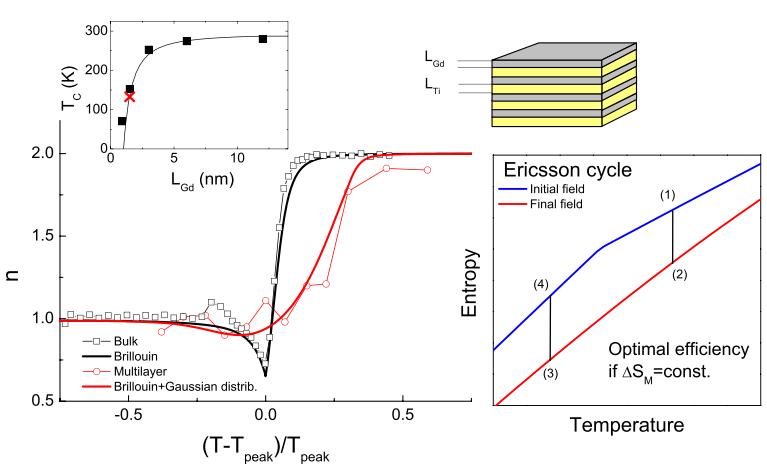

\begin{abstract}
A B S T R A C T
In this work, the field dependence of the magnetocaloric effect of Gd bulk samples has been enhanced through nanostructuring of the material. Nanostructuring consists in multilayers preparation by alternative rf-sputtering deposition of Gd layers and Ti spacers onto glass substrates. The results obtained for the multilayers were compared to those obtained for the Gd bulk. Assuming a power law for the field dependence of the magnetic entropy change $\left(\Delta S_{M} \propto H^{n}\right)$, higher field dependences close to the transition in a wider temperature range are obtained for the multilayer material $(n=1.0)$ with respect to the bulk counterpart $(n=0.78)$. The effect of a Curie temperature distribution in the multilayer material (due to variations of the layer thickness) has been studied through numerical simulations to explain the observed field dependence of the magnetocaloric effect, obtaining a remarkable agreement between experiments and results.
\end{abstract}

(c) 2016 Elsevier Ltd. All rights reserved.

\footnotetext{
* Corresponding author.

E-mail address: vfranco@us.es (V. Franco).

1 Current address: Leibniz-Institut für Neue Materialien, Campus D2 2, 66123 Saarbrücken, Germany.
}

\section{Introduction}

The magnetocaloric effect (MCE), i.e. the reversible temperature change of a magnetic material upon the application/removal of a magnetic field, is routinely used in research laboratories to achieve ultralow temperatures. This effect is maximum when the material exhibits 
an abrupt change of magnetization with temperature. For ultra-low temperatures, paramagnetic salts are typically used, as they exhibit a noticeable temperature change of their magnetic properties close to $0 \mathrm{~K}$. On the other hand, there is a possibility to employ this effect at room temperature in magnetic refrigerators. Although magnetic refrigeration is not a commercial technology yet, current prototypes have shown that energy efficiency can be increased by more than $50 \%$ with respect to the optimal refrigerators based on the compression/expansion of gasses. Adding to the equation the absence of gasses responsible for greenhouse effect and ozone depletion, the reduced vibration and noise motivated by the lack of a large compressor, and the operation at any tilt angle, one finds the arguments which support research in magnetocaloric materials $[1,2]$.

The archetypical magnetocaloric material at room temperature is Gd, since Brown proposed its application for this purpose in 1974 [3], due to its large magnetic moment and the second order magnetic phase transition close to room temperature. Subsequently, the discovery of the giant magnetocaloric effect in GdSiGe [4] shifted the interest to materials with a first order magneto-structural phase transition. This latter kind of materials exhibit a larger MCE response but it has inherent thermal hysteresis due to the nature of the phase transition, which complicates its applicability in current prototypes. At the moment, most magnetic refrigerators used either Gd, LaFeSiH or MnFePSi as refrigerant materials, although the second material has the drawbacks of mechanical degradation after magnetic field cycling and splitting of the magnetic entropy change peaks due to instability of the hydride phase, and the two latter present hysteresis, issues which are driving further research to minimize them [5-7]. The most recent demonstrator of a domestic appliance that is close to commercialization has been presented by Haier, in cooperation with Astronautics Corporation of America, BASF and Delft University of Technology in CES Las Vegas 2015.

For all temperature ranges, the magnetocaloric response of a material increases as the magnetic field increases. Therefore, a way of improving the response of the refrigerant material in a refrigerator device would be to apply larger magnetic fields. However, current refrigerator designs are limited by the field values which can be applied by permanent magnets and prototypes use fields in the range of 0.8-1.5 T. Increasing the field above these values would complicate the design of the device and the associated cost. Therefore, finding and understanding methods for increasing the magnetic field responsiveness of magnetocaloric materials could eventually lead to newer possibilities in the application of magnetic refrigeration.

There have been previous reports on the different magnetocaloric response of materials with polycrystalline and nanocrystalline microstructures [8-10]. For example, in the case of manganites [11], polycrystalline samples have a magnetocaloric response which is spread over a narrow temperature range, producing a relatively large magnetic entropy change peak. However, structurally inhomogeneous nanocrystalline compounds of the same nominal composition have a much broader peak. There are also increasing efforts for the preparation of both conventional $[12,13]$ and giant magnetocaloric materials in thin film form [14-18].

Nanostructuring can also alter the magnetocaloric behavior of Fe-Rh, as its reversibility in enhanced when fabricated in the form of thin films and submitted to a multicaloric cycle [19]. Size effects are also known to affect Heusler alloys, as they presumably broaden the martensitic transformation and decrease the transformation temperatures of thin films of NiMnSn [20].

The aim of this work is to demonstrate that nanostructuring is a promising way of enhancing the rate of change of the magnetocaloric response with field. It has been shown [21] that FeNi electrodeposited multilayers with different (graded) composition of each of the layers lead to a linear field dependence of the magnetic entropy change peak $\left(\Delta S_{M}^{p k}\right)$, which is a larger field responsiveness than the typical of bulk materials. In that case, FeNi was chosen due to the facility of controlling the composition of the layers (which in turn controls the Curie temperature, $T_{C}$, of each of them) and the possibility of fabricating it by electrodeposition. However, the response of the multilayer system was small, partially inherited from the small magnetocaloric response of bulk FeNi. The presence of a distribution of Curie temperatures in the sample also produces a broader peak of the magnetocaloric effect. And although this is connected to a decrease in the magnitude of the peak, a table-like magnetocaloric effect could be beneficial for Ericsson type refrigeration cycles [22-26]. In fact, Ericsson-like AMR cycle makes use of isothermal magnetization and demagnetization steps. In order to be close to these conditions, there should be an appropriate fluid flow rate. However, as most materials exhibit a strong temperature dependence of the magnetocaloric response, it is practically impossible to ensure an isothermal process with a flow rate which is not dependent on position along the whole length of the AMR bed. In the case that the magnetocaloric material exhibits a table-like response, i.e. not depending strongly on temperature, this limitation can be alleviated.

In this work we succeed to demonstrate that finite size scaling in Gd/ Ti multilayers prepared by sputtering allows to obtain multilayered materials with a single composition of the layers yet exhibiting a linear field dependence due to thickness layer variations. In addition, although nanostructuring usually decreases the magnitude of the magnetocaloric response of materials with respect to their bulk counterpart [27], the experimental evidences shown in this work can be used for finding the ways to enhance the magnetic field responsiveness of magnetocaloric materials used in future prototypes.

\section{Experimental}

The $[\operatorname{Ti}(2 \mathrm{~nm}) / \mathrm{Gd}(1.5 \mathrm{~nm})]_{50} / \operatorname{Ti}(5 \mathrm{~nm})$ multilayers were prepared by alternative rf-sputtering deposition of Gd layers and Ti spacers onto glass substrates. The base pressure in the chamber was $3 \times 10^{-7} \mathrm{mbar}$ and argon gas flow with a pressure of $5 \times 10^{-3}$ mbar was used for the sputtering process. The deposition rate was about $0.15 \mathrm{~nm} / \mathrm{s}$ for $\mathrm{Gd}$ and about $0.07 \mathrm{~nm} / \mathrm{s}$ for Ti. The thicknesses of the Gd layers $\left(\mathrm{L}_{\mathrm{Gd}}\right)$ was chosen to be $1.5 \mathrm{~nm}$ in order to be close to the values for which a small change of thickness would produce a large change of Curie temperature, as will be seen in Section 3.1. Ti was chosen for the spacer as a material with low solubility in rare earth materials. In a previous work it was shown that Ti buffer layer of $2 \mathrm{~nm}$ completely interrupts the magnetic interaction between Gd layers [28]. Therefore, in order to rule out the effect of interactions on the magnetocaloric response, we have selected that thickness for the buffer. The sample has a protective coating $\operatorname{Ti}(5 \mathrm{~nm})$ layer. The microstructure of the samples was examined by transmission electron microscopy (TEM) and X-ray diffraction (XRD). Further details about fabrication and structural characterization of the sample can be found elsewhere [28]. Magnetic measurements were performed in a LakeShore 7407 vibrating sample magnetometer and MCE analysis has been done with the help of the magnetocaloric effect analysis program [29]. The mass of the magnetic layers of the multilayered sample was calculated from the thickness of the layers, the area of the sample and the density of bulk Gd. Even if the density of the thin Gd layers would be smaller than that of bulk $\mathrm{Gd}$, the error in calculating the mass in this way is much smaller than that of weighting the sample in a balance, due to the fact that the substrate has a much larger mass than the sample. This error produces an underestimated value of the magnetic entropy change, but does not alter its field or temperature dependences. Results for the multilayers are compared with the behavior of commercial crystalline Gd $(99.9 \%$ purity), which was melt spun in order to obtain ribbon shaped samples, $\sim 20 \mu \mathrm{m}$ thick and $\sim 3 \mathrm{~mm}$ wide (although the ratio of sizes is large, the demagnetizing factor when measured with the field applied in the plane of the ribbon is not zero due to surface roughness, pores, etc. of the polycrystalline sample). 


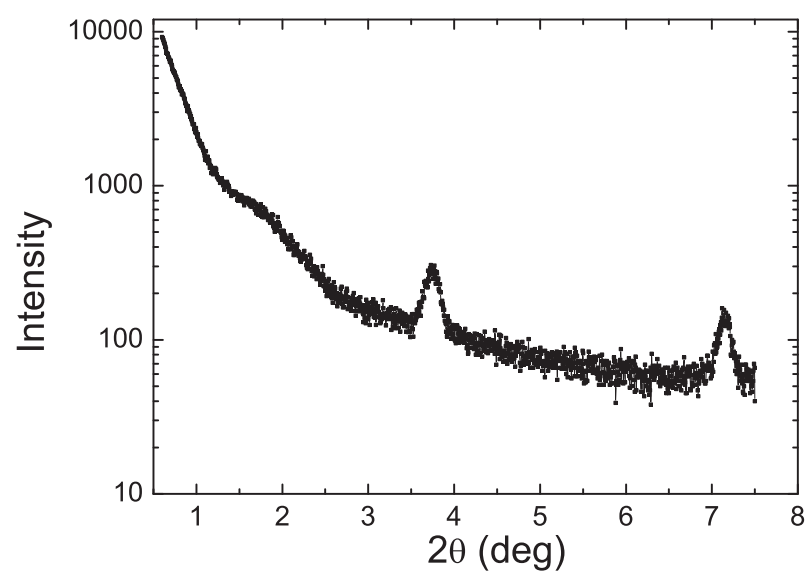

Fig. 1. Low-angle X-ray diffractogram for the Gd/Ti multilayer sample.

\section{Results}

\subsection{Curie temperature of magnetic multilayers}

In a multilayer material due to finite size scaling, the value of $T_{C}$ is related with the thickness of the layer $(L)$ by a power law [30]:

$\frac{T_{C}(\text { bulk })-T_{C}}{T_{C}(\text { bulk })}=C L^{-\lambda}$

where $T_{C}$ (bulk) is the Curie temperature for the bulk material and $C$ and $\lambda$ are constant.

Fig. 1 shows the low-angle $\mathrm{X}$ ray diffractogram for the $\mathrm{Gd} / \mathrm{Ti}$ multilayer sample. The observed peaks allow the determination of the layer thickness, which agrees quite well with that expected from the deposition time. The periodic structure was determined by using low angle Xray diffraction, which was carried out on a Rigaku $D /$ max-RB X-ray diffractometer using a rotating-anode $\mathrm{Cu} \mathrm{K}_{\alpha}$ source $(\lambda=0.154 \mathrm{~nm})$. The multilayer period d can be accurately determined by a modified Bragg equation

$$
\sin \theta=m \lambda / 2 d
$$

where $\theta$ is the angular positions of the peaks, $m$ is the reflection order number, $\lambda$ is the radiation wavelength. However, X-ray diffraction might not be the most precise method for the determination of the absolute value of the periodicity in the multilayered structures, especially for the very thin period value when small number of peaks is present, like in this case. This might be the reason for the lower value for the sample period in comparison with the thickness expected from the deposition rate calculating the thickness from the layers' deposition time (Table 1). However, in the present work the absolute value of the thickness of the layers is not so important comparing to the fact of the existence the well-defined layered structure. The last is evidenced by the presence of well-defined peaks at a low angle X-ray diffractograms. Well defined layers are also visible in the cross-sectional HRTEM image obtained by means of JEOL JEM-2100 (Fig. 2). High angle X-ray diffractograms did not contain peaks. Instead, an amorphous-like halo

Table 1

The bilayer thickness determined from the reflection of different order for $[\mathrm{Gd}(1.5 \mathrm{~nm}) / \mathrm{Ti}(2 \mathrm{~nm})]_{50}$ sample.

\begin{tabular}{ll}
\hline Peak order & Corresponding bilayer thickness $(\mathrm{nm})$ \\
\hline 1 & 2.4 \\
2 & 2.5 \\
\hline
\end{tabular}

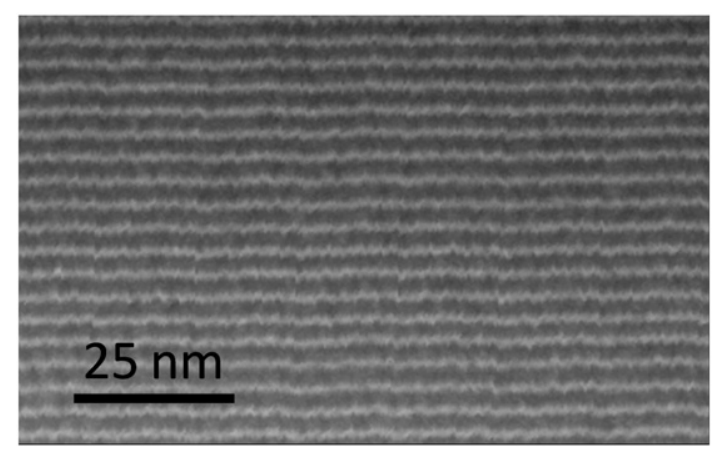

Fig. 2. Cross-sectional HRTEM image for $[\mathrm{Gd}(1.5 \mathrm{~nm}) / \mathrm{Ti}(2 \mathrm{~nm})]_{50}$ sample.

was observed. This confirms the existence of an amorphous structure of the layers.

Fig. 3 shows the temperature dependence of the magnetization at low field (120 Oe) for the Gd/Ti multilayer sample. The Curie temperature is estimated as the inflection point of this magnetization curve ( $T_{C} \approx 130 \mathrm{~K}$ ). Inset of Fig. 3 shows the dependence of $T_{C}$ with respect to the thickness for Gd/Ti multilayers (fitted to Eq. (1)) using $L$ as the thickness of the Gd layers. The estimated value of the thickness is around $1.5 \mathrm{~nm}$, in agreement with the obtained value by low-angle XRD.

\subsection{Isothermal magnetization curves}

Figs. 4 and 5 show the magnetization curves measured at constant temperature for applied fields up to $1.5 \mathrm{~T}$ for the Gd bulk material and $\mathrm{Gd} / \mathrm{Ti}$ multilayer, respectively. For the bulk sample, the response of the sample holder is negligible. However, in the case of the multilayers, the total measured magnetic moment contains the response of the $\mathrm{Gd} / \mathrm{Ti}$ multilayers plus the response of the sample holder and glass substrate. In order to be able to deconvolute that signal, it has been measured and plotted as a dashed line in Fig. 5. As this additional signal does not exhibit a noticeable temperature dependence, the calculation of the magnetocaloric response from the raw magnetization data is not affected and it does not need to be subtracted.

\subsection{Magnetocaloric response}

Figs. 6 and 7 show the magnetic entropy change $\left(\Delta S_{M}\right)$ for the Gd bulk material and $\mathrm{Gd} / \mathrm{Ti}$ multilayer, respectively. If we compare both curves, it can be observed that the bulk sample has a higher value of $\Delta S_{M}$ peak $\left(\Delta S_{M}^{p k}\right)$ than the multilayer one (at $1.5 \mathrm{~T}, \Delta S_{M}^{p k}$ is -3.5 and $-1 \mathrm{~J} \mathrm{~kg}^{-1} \mathrm{~K}^{-1}$ for the bulk and multilayer samples, respectively). This

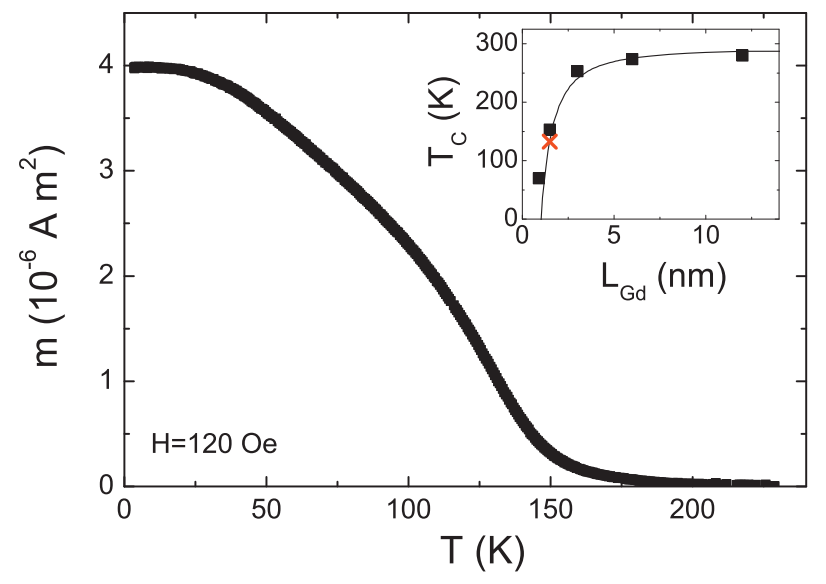

Fig. 3. Magnetization curve at 120 Oe for the $\mathrm{Gd} / \mathrm{Ti}$ multilayer sample. Inset: Curie temperature evolution with respect the layer thickness [28]. 


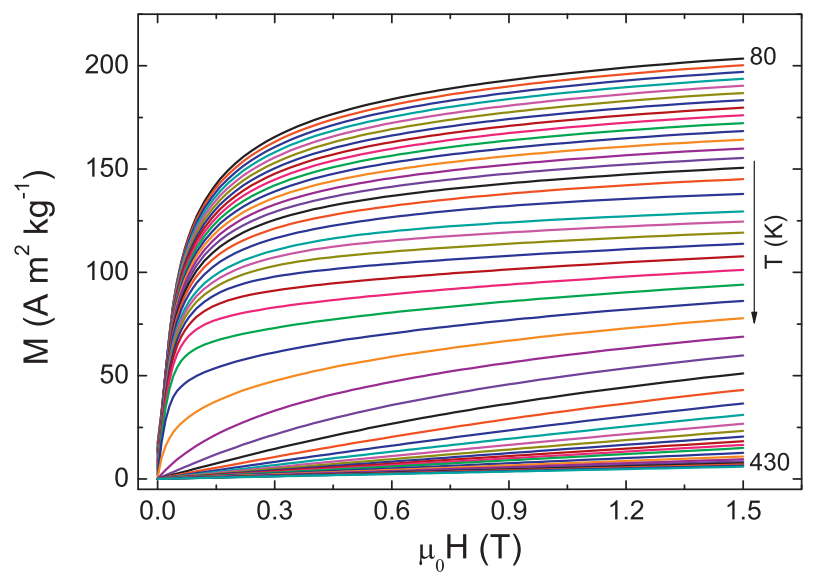

Fig. 4. Field dependent magnetization of the bulk Gd sample measured at different temperatures.

fact is usually observed in nanostructured materials with respect to their bulk counterparts [27]. On the other hand, the $\Delta S_{M}$ curves for the multilayer material are wider than those of the bulk.

It was shown, for a magnetocaloric material with a second order phase transition, that a power law of the form $\Delta S_{M} \propto H^{n}$ can be used to study the field dependence of the magnetic entropy change [31]. For a single phase material, the exponent $n$ is field independent in three temperature regimes: well above $T_{C}(n=2)$, well below $T_{C}(n=1)$ and at $T_{C}$. The value of $n$ at $T_{C}$ is related to the critical exponents [31] of the material, $n=1+(1-1 / \beta) / \delta$, where $\beta$ and $\delta$ are the critical exponents with their usual meaning [32]. For the mean field case $n=2 / 3$. It is interesting to note that $\Delta S_{M}$ responds more to the field in those temperature regions where its value is small (fully ferromagnetic and fully paramagnetic ranges), having the lowest field responsiveness close to the Curie temperature, where delta $\Delta S_{M}$ has its peak.

An increase of this exponent $n$ in the region close to $T_{C}$ can improve the applicability of the magnetocaloric materials as it would allow us to get higher response for the same applied field. Fig. 8 shows the evolution of $\Delta S_{M}^{p k}$ with respect to the magnetic field (in a log-log plot) for the Gd/Ti multilayer and Gd bulk samples. The value of the exponent $n$ for the bulk Gd is $0.78(1)$, while for the Gd/Ti multilayer the value increases to 1.01(1). At low fields the field dependence deviates from the expected linear behavior in log-log plots due to the multidomain structure of the sample (theoretical predictions of the scaling laws are only valid in the case of technically saturated samples). Above $0.5 \mathrm{~T}$ fields,

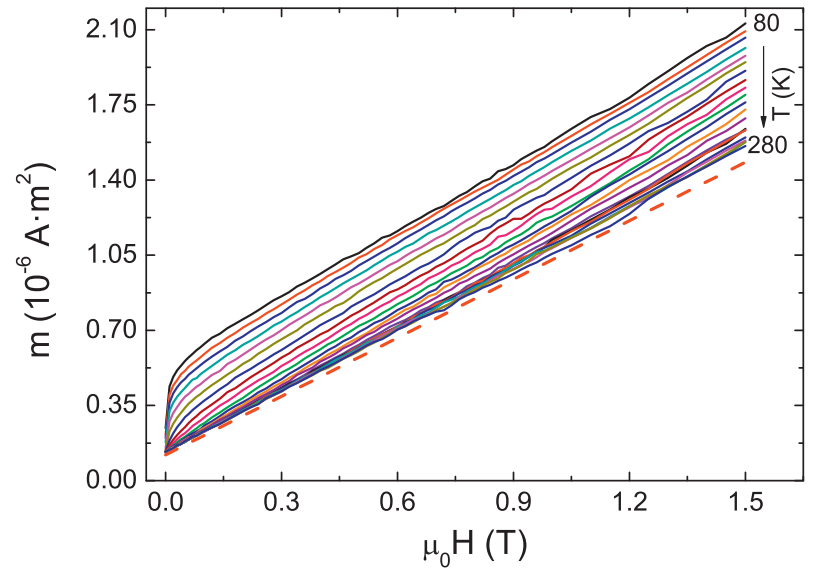

Fig. 5. Field dependence magnetization of the $\mathrm{Gd} / \mathrm{Ti}$ multilayer sample measured at different temperatures. Dashed line corresponds to the temperature independent magnetic contribution of the sample holder + blank substrate.

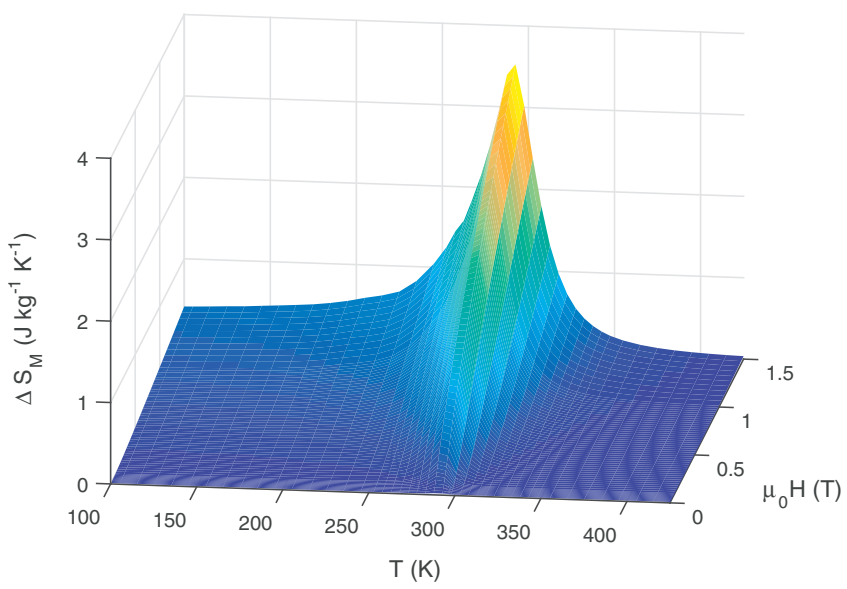

Fig. 6. Magnetic entropy change for the Gd bulk sample.

where the samples reach the technical saturation state, the fitting to a power law can be done.

In order to compare the temperature dependence of the exponent $n$ for the multilayer and bulk samples, it is necessary to rescale the temperature axis, as the temperature of $\Delta S_{M}^{p k}$ is different in both cases. Fig. 9 shows the local exponent $n$ (obtained from $n=\operatorname{dlog}\left(\Delta S_{M}\right) / \operatorname{dlog}(H)$ instead of obtaining it from a linear fit in a log-log plot) with respect to the reduced temperature, $\left(T-T_{C}\right) / T_{C}$, for both samples at $1.5 \mathrm{~T}$. The local exponent $n$ of the multilayer material is around $1( \pm 0.1)$ in the region close to $T_{C}$ (as it was observed before in Fig. 8) and it is constant in a wide temperature span around $T_{C}$. These results are similar to those obtained for $\mathrm{Ni} / \mathrm{Cu}$ multilayers with different composition in the layers [21], but with the advantage, in our case, that this effect is achieved with a single composition. Concerning the bulk sample, the experimental data have to be corrected making use of an effective demagnetizing factor, which could be ascribed to different defects (e.g. inhomogeneities, oxides, surface irregularities, etc.). For the bulk Gd sample, in addition to the previously described temperature evolution of $n$, an additional peak can be observed around $235 \mathrm{~K}$ which is ascribed to spin reorientations in Gd [33].

The wider and smaller peak of the magnetocaloric response curve for the $\mathrm{Gd} / \mathrm{Ti}$ multilayer material can be ascribed to a distribution of Curie temperatures due to a distribution of layer thicknesses. To check this claim, numerical simulations have been performed. Magnetization of the Gd bulk sample has been modelled through the Brillouin equation of state (that assumes a mean field theory). In order to model the

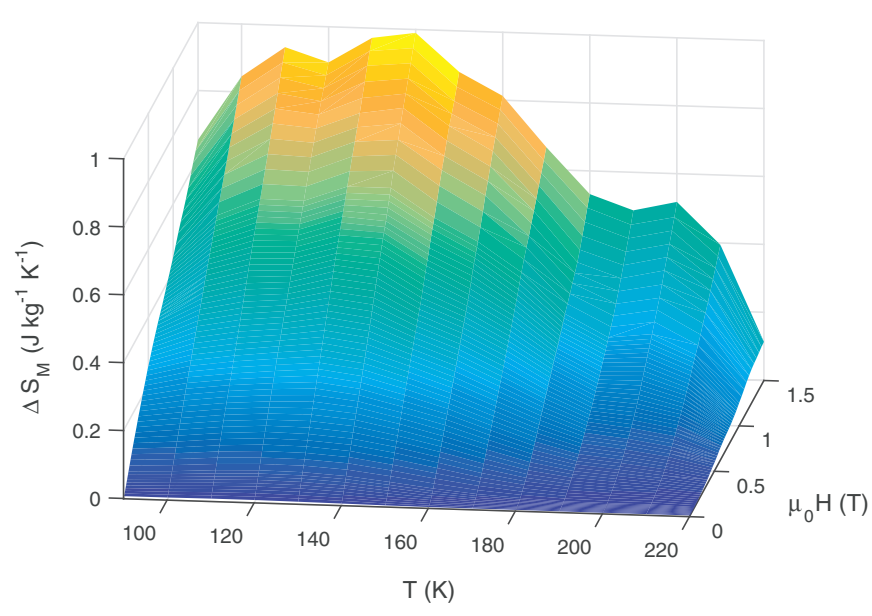

Fig. 7. Magnetic entropy change for the Gd/Ti multilayer sample. 


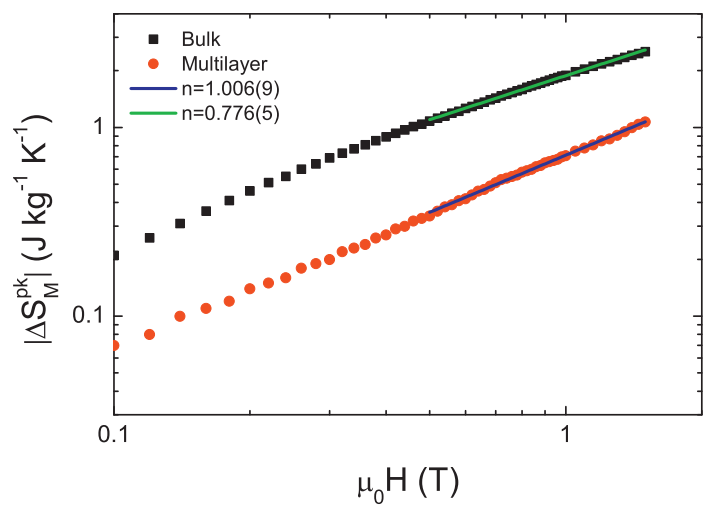

Fig. 8. Field evolution of the magnetic entropy change peak for the multilayer $\mathrm{Gd} / \mathrm{Ti}$ and bulk Gd samples.

behavior of the multilayer material, the previous bulk behavior has been modified through a Gaussian distribution of $T_{C}$ values. Therefore, the $\Delta S_{M}(T)$ for the $\mathrm{Gd} / \mathrm{Ti}$ multilayer sample can be expressed as:

$\Delta S_{M}(T)=C \int_{T_{C}-\delta}^{T_{C}+\delta} \Delta S_{M}^{B r i}\left(T, T_{C}^{\prime}\right) \exp \left(-\left(T_{C}^{\prime}-T_{C}\right)^{2} / 2 \sigma^{2}\right) d T_{C^{\prime}}$

where $\sigma$ is the standard deviation, $2 \delta$ is the range of the distribution of Curie temperatures and $C$ is the normalization constant. The inset of Fig. 6 shows the calculated values of $n$ at $T_{C}$ as a function of $\sigma$. It can be observed that the values of the exponent $n$ increases as $\sigma$ increases, reaching values close to the observed ones at $\sigma \approx 30 \mathrm{~K}$. The distribution of $T_{C}$ can be ascribed to small differences in the layer thickness. In fact, adding or subtracting one atomic monolayer leads to a variation of the $T_{C}$ around $100 \mathrm{~K}$ as can be seen in the inset of Fig. 3. Surface defects (e.g. steps, islands, etc.) could also play a role. The obtained values of $n$

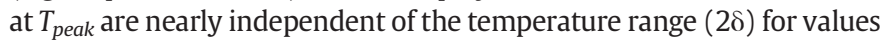
close to $\delta=2 \sigma$ (the integrand for $T_{C}{ }^{\prime}>T_{C}+2 \sigma$ is negligible). The assumption of a $\sigma \approx 30 \mathrm{~K}$ implies a temperature range for the distribution of the same order than those produced by one atomic layer change. Fig. 9 shows the temperature dependence for the simulated data in which a qualitative agreement with respect to the experimental data is observed.

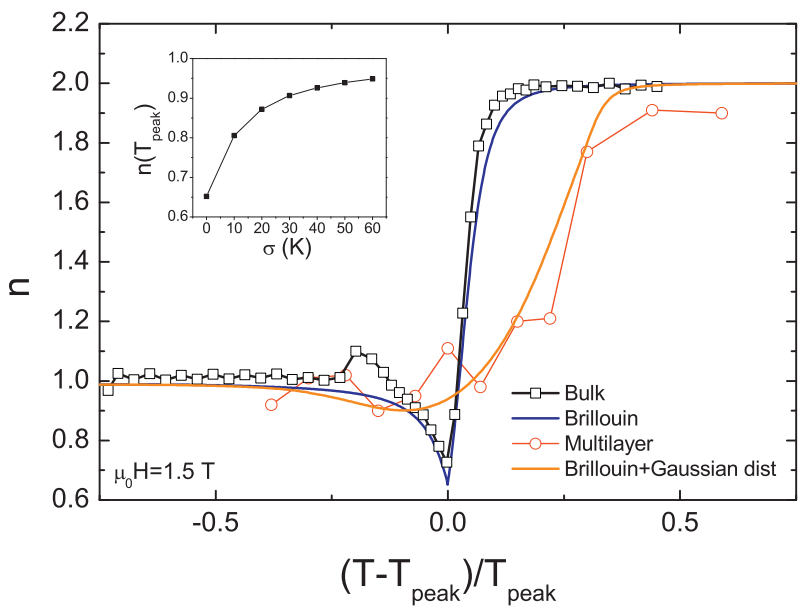

Fig. 9. Temperature dependence of the exponent $\mathrm{n}$ at $15 \mathrm{kOe}$ of the $\mathrm{Gd} / \mathrm{Ti}$ multilayer and Gd bulk samples and numerical simulations using the Brillouin equation of state and assuming a Gaussian distribution of Curie temperatures $(\sigma=30 \mathrm{~K})$. Inset: Evolution of the exponent $n$ at $T_{\text {peak }}$ with respect the sigma parameter of the distribution of Curie temperatures.

\section{Conclusions}

The field dependence of the magnetocaloric effect for Gd samples has been modified through nanostructuring the material ( $\mathrm{Gd} / \mathrm{Ti}$ multilayers of $1.5 \mathrm{~nm}$ thickness). Higher values of the exponent $n$ at the transition temperature and in a wider temperature range were obtained for the multilayer material with respect to the bulk. This fact can be ascribed to a Curie temperature distribution due to tiny variations in the layer thickness (including surface defects) of the order of one atomic monolayer. This has been proven by numerical simulations in which the multilayer material has been reproduced using the Brillouin equation of state to describe each contribution of a Gaussian distribution of Curie temperatures. As the application of magnetic field is an expensive part of magnetic refrigerator prototypes, the relevance of this work is to show a possible method to increase the magnetic field responsiveness of magnetocaloric materials close to the peak of the transition.

\section{Acknowledgements}

This work was supported by the Spanish MINECO and EU-FEDER (projects MAT2013-45165-P and MAT2016-77265-R), the PAI of the Regional Government of Andalucía, and the Ministry of Education and Science of the Russian Federation (Project No. 2582). L.M. MorenoRamírez acknowledges a FPU fellowship from the Spanish MECD. Selected measurements were performed at SGIker service of UPV-EHU. We thank A. Larrañaga for excellent technical support.

\section{References}

[1] O. Gutfleisch, M.A. Willard, E. Bruck, C.H. Chen, S.G. Sankar, J.P. Liu, Magnetic materials and devices for the 21st century: stronger, lighter, and more energy efficient, Adv. Mater. 23 (2011) 821-842.

[2] K.A. Gschneidner, V.K. Pecharsky, Magnetocaloric materials, Annu. Rev. Mater. Sci. 30 (2000) 387-429.

[3] G.V. Brown, Magnetic heat pumping near room temperature, J. Appl. Phys. 47 (1976) 3673-3680.

[4] V.K. Pecharsky, K.A. Gschneidner, Giant magnetocaloric effect in $\mathrm{Gd}_{5}\left(\mathrm{Si}_{2} \mathrm{Ge}_{2}\right)$, Phys Rev. Lett. 78 (1997) 4494-4497.

[5] J. Lyubina, R. Schafer, N. Martin, L. Schultz, O. Gutfleisch, Novel design of La (Fe, Si) 13 alloys towards high magnetic refrigeration performance, Adv. Mater. 22 (2010) 3735-3739.

[6] J. Lyubina, U. Hannemann, M.P. Ryan, L.F. Cohen, Electrolytic Hydriding of LaFe $\mathrm{L}^{-}$ $\mathrm{XSi}_{\mathrm{x}}$ alloys for energy efficient magnetic cooling, Adv. Mater. 24 (2012) 2042-2046.

[7] F. Guillou, H. Yibole, G. Porcari, L. Zhang, N.H. van Dijk, E. Brück, Magnetocaloric effect, cyclability and coefficient of refrigerant performance in the $\mathrm{MnFe}(\mathrm{P}, \mathrm{Si}, \mathrm{B})$ system, J. Appl. Phys. 116 (2014) 063903.

[8] S. Ma, D.Y. Geng, W.S. Zhang, W. Liu, X.L. Ma, Z.D. Zhang, Synthesis of a new type of $\mathrm{GdAl}_{2}$ nanocapsule with a large cryogenic magnetocaloric effect and novel coral-like aggregates self-assembled by nanocapsules, Nanotechnology 17 (2006) 5406.

[9] S.P. Mathew, S.N. Kaul, Tuning magnetocaloric effect with nanocrystallite size, Appl. Phys. Lett. 98 (2011) 172505.

[10] P. Lampen, N.S. Bingham, M.H. Phan, H. Kim, M. Osofsky, A. Pique, T.L. Phan, S.C. Yu, H. Srikanth, Impact of reduced dimensionality on the magnetic and magnetocaloric response of La0·7Ca0.3MnO3, Appl. Phys. Lett. 102 (2013) 062414.

[11] M. Pekala, V. Drozd, Magnetocaloric effect in nano- and polycrystalline $\mathrm{La}_{0.8} \mathrm{Sr}_{0.2} \mathrm{MnO}_{3}$ manganites, J. Non-Cryst. Solids 354 (2008) 5308-5314.

[12] F. Hellman, E.N. Abarra, A.L. Shapiro, R.B. van Dover, Specific heat of amorphous rare-earth-transition-metal films, Phys. Rev. B 58 (1998) 5672.

[13] H.F. Kirby, D.D. Belyea, J.T. Willman, C.W. Miller, Effects of preparation conditions on the magnetocaloric properties of Gd thin films, J. Vac. Sci. Technol. A 31 (2013).

[14] V. Recarte, J.I. Perez-Landazabal, V. Sanchez-Alarcos, V.A. Chernenko, M. Ohtsuka, Magnetocaloric effect linked to the martensitic transformation in sputter-deposited Ni-Mn-Ga thin films, Appl. Phys. Lett. 95 (2009) 141908.

[15] R. Niemann, L. Schultz, S. Fahler, Growth of sputter-deposited metamagnetic epitaxial Ni-Co-Mn-In films, J. Appl. Phys. 111 (2012) 093909.

[16] R.L. Hadimani, I.C. Nlebedim, Y. Melikhov, D.C. Jiles, Growth and characterisation of Gd-5 $\left(\mathrm{Si}_{\mathrm{x}} \mathrm{Ge}_{1}-\mathrm{x}\right)(4)$ thin film, J. Appl. Phys. 113 (2013).

[17] Yuzuak, I. Dincer, Y. Elerman, A. Auge, N. Teichert, A. Hutten, Inverse magnetocaloric effect of epitaxial Ni-Mn-Sn thin films, Appl. Phys. Lett. 103 (222403) (2013).

[18] R.L. Hadimani, Y. Mudryk, T.E. Prost, V.K. Pecharsky, K.A. Gschneidner, D.C. Jiles, Growth and characterization of Pt-protected $\mathrm{Gd}_{5} \mathrm{Si}_{4}$ thin films, J. Appl. Phys. 115 (2014) 17C113.

[19] Y. Liu, L.C. Phillips, R. Mattana, M. Bibes, A. Barthelemy, B. Dkhil, Large reversible caloric effect in FeRh thin films via a dual-stimulus multicaloric cycle, Nat. Commun. 7 (2016) 11614.

[20] N. Teichert, A. Auge, E. Yuzuak, I. Dincer, Y. Elerman, B. Krumme, H. Wende, O. Yildirim, K. Potzger, A. Hutten, Influence of film thickness and composition on the 
martensitic transformation in epitaxial Ni-Mn-Sn thin films, Acta Mater. 86 (2015) 279.

[21] R. Caballero-Flores, V. Franco, A. Conde, L.F. Kiss, L. Péter, I. Bakonyi, Magnetic multilayers as a way to increase the magnetic field responsiveness of magnetocaloric materials, J. Nanosci. Nanotechnol. 12 (2012) 7432-7436.

[22] A. Kitanovski, J. Tušek, U. Tomc, U. Plaznik, M. Ozbolt, A. Poredoš, Magnetocaloric Energy Conversion: From Theory to Applications, Springer, Cham, 2015105

[23] Z. Ma, Y.F. Shang, E. Agurgo Balfour, H. Fu, B.H. Teng, L. Wang, Y. Luo, S.F. Wang, Y.H Wu, M.G. Han, Comparative study of the magnetocaloric effect in multiphase GdNiA alloys: single peak versus table-like profile in magnetic entropy changes, J. Alloys Compd. 680 (2016) 268-272.

[24] M.V. de Souza, Investigation on the magnetocaloric effect in $\mathrm{RNi}_{2}$ (R: Dy, tb) meltspun ribbon, J. Magn. Magn. Mater. 412 (2016) 11-14.

[25] R. M'nassri, N. Chniba Boudjada, A. Cheikhrouhou, Nearly constant magnetic entropy change involving the enhancement of refrigerant capacity in $\left(\mathrm{La}_{06} \mathrm{Ba}_{02} \mathrm{Sr}_{02} \mathrm{MnO}_{3}\right)_{1-x}$ $\left(\mathrm{Co}_{2} \mathrm{O}_{3}\right)_{\mathrm{x}}$ composite, Ceram. Int. 42 (2016) 7447-7454.

[26] G.L. Liu, D.Q. Zhao, H.Y. Bai, W.H. Wang, M.X. Pan, Room temperature table-like magnetocaloric effect in amorphous $\mathrm{Gd}_{50} \mathrm{Co}_{45} \mathrm{Fe}_{5}$ ribbon, J. Phys. D. Appl. Phys. 49 (2016) 055004.
[27] V. Franco, A. Conde, Magnetic refrigerants with continuous phase transitions: amorphous and nanostructured materials, Scr. Mater. 67 (2012) 594-599.

[28] A.V. Svalov, V.O. Vas'kovskiy, J.M. Barandiaran, K.G. Balymov, A.N. Sorokin, I. Orue, A. Larrañaga, N.N. Schegoleva, G.V. Kurlyandskaya, Structure and magnetic properties of Gd/Ti nanoscale multilayers, Solid State Phenom. 168-169 (2011) 281-284.

[29] V. Franco, B.C. Dodrill, C. Radu, Magnetocaloric measurements: from energy efficient refrigeration to a tool for the study of phase transitions, Manuf. Bus. Technol. 13 (2014) 8http://www.lakeshore.com/products/Vibrating-Sample-Magnetometer/ Pages/MCE.aspx.

[30] M. Farle, K. Baberschke, U. Stetter, A. Aspelmeier, F. Gerhardter, Thickness-dependent curie temperature of $\mathrm{Gd}(0001) / \mathrm{W}(110)$ and its dependence on the growth conditions, Phys. Rev. B 47 (1993) 11571-11574.

[31] V. Franco, A. Conde, Scaling laws for the magnetocaloric effect in second order phase transitions: from physics to applications for the characterization of materials, Int. J. Refrig. 33 (2010) 465-473.

[32] R.B. Griffiths, Thermodynamic functions for fluids and ferromagnets near the critical point, Phys. Rev. 158 (1967) 176-187.

[33] R.E. Camley, Surface spin reorientation in thin Gd films on Fe in an applied magnetic field, Phys. Rev. B 35 (1987) 3608-3611. 